Koichi Fukino • Akira Teramoto $\cdot$ Koji Adachi

Hiroshi Takahashi $\cdot$ Mitsuru Emi

\title{
A family with hydrocephalus as a complication of cerebellar hemangioblastoma: identification of Pro157Leu mutation in the VHL gene
}

\begin{abstract}
Various mutations in the $V H L$ gene on chromosome 3p25-26 are responsible for von Hippel-Lindau (VHL) syndrome. We report on a Japanese VHL family in which two of the three affected members developed acute occlusive hydrocephalus that necessitated emergency surgery for ventricular shunt or drainage. Direct sequencing and restriction fragment length polymorphism analysis identified a germline missense mutation, Proline-toLeucine, caused by a C-to-T transition at the second nucleotide of codon 157.
\end{abstract}

Key words von Hippel-Lindau disease . $V H L$ gene . germline mutation - Restriction fragment length polymorphism $\cdot$ Hemangioblastoma

\section{Introduction}

von Hippel-Lindau (VHL) syndrome is a heritable neoplastic disorder characterized by susceptibility to cerebellar and spinal hemangioblastomas, retinal angiomas, renal cell carcinomas, pheochromocytomas, and cysts in the pancreas, kidneys, and other organs (Melmon and Rosen 1964). This disease occurs in families of many ethnic backgrounds (Kley et al. 1995).

The gene responsible for VHL syndrome lies in chromosome band 3p25-26. Various mutations have been documented in this gene, whose product inhibits transcription elongation by binding to a cellular transcription factor, Elongin (Tory et al. 1989; Hosoe et al. 1990; Maher et al. 1991; Seizinger et al. 1991; Yao et al. 1993; Crossey et al.

K. Fukino $\cdot$ M. Emi $(\square)$

Department of Molecular Biology, Institute of Gerontology, Nippon Medical School, 1-396 Kosugi-cho, Nakahara-ku, Kawasaki-shi 211-8533, Japan

Tel. +81-44-733-5230; Fax +81-44-733-5192

e-mail:memi@nms.ac.jp

K. Fukino $\cdot$ A. Teramoto $\cdot$ K. Adachi $\cdot$ H. Takahashi

Department of Neurosurgery, Nippon Medical School, Tokyo, Japan
1993 and 1994a,b; Clinical Research Group for VHL in Japan 1995; Duan et al. 1995; Kibel et al. 1995).

Heterogeneity of phenotypes is a salient feature of VHL syndrome, and the disease has been classified into at least two clinical entities: VHL type 1 does not include pheochromocytomas in its phenotype, and VHL type 2 does (Chen et al. 1995; Glavac et al. 1996). In spite of the variety of manifestations within both categories, the clinical courses of VHL patients are usually chronic in nature. However, occasionally, some patients manifest the disease as lifethreatening hydrocephalus, an unusual form of onset that results from occlusion of the spinocerebral canal by a cerebellar hemangioblastoma.

We report here on a Japanese nuclear family in which two of the three members affected with VHL developed acute occlusive hydrocephalus that necessitated emergency surgery for ventricular shunt or drainage.

\section{Clinical report}

Patient 1

This patient was the older daughter of the family. At age 8 years she was admitted to our hospital presenting with headache, vomiting, gait disturbance, and diplopia; neurological examination revealed cerebellar signs, including truncal ataxic gait, dysmetria, and nystagmus. Computed tomography (CT) scan of her head indicated obstructive hydrocephalus caused by a cerebellar tumor, and she underwent emergency surgery for a ventriculo-peritoneal shunt. After 2 weeks, the cerebellar tumor was removed for the first time, and histologically diagnosed as a hemangioblastoma; she was immediately treated with irradiation to the head (20 Gy). At ages 15 and 19, she underwent second and the third removals of the recurring hemangioblastoma. At the time of the third surgery she was suffering visual loss caused by retinal angiomas of the left eye, and an abdominal CT scan revealed that she also had a cystic lesion in the pancreas. These symptoms led to a clinical diagnosis of von 
Hippel-Lindau disease. She has since become blind in the left eye and has hypoacusis in the left ear.

\section{Patient 2}

This patient was the mother of patients 1 and 3. At age 39, suffering from retinal angiomas and a cerebellar hemangioblastoma, she underwent surgery for removal of the brain tumor in another hospital. After the operation she suffered from left facial palsy.

\section{Patient 3}

Patient 3 was the younger sister of patient 1 . In view of her family history of von Hippel-Lindau disease, she underwent head CT and magnetic resonance imaging (MRI) at age 14 to determine whether she had developed any intracranial tumors. The MRI showed at least three (Fig. 1A), but no surgical treatment was performed at this time because the tumors were very small. Five years later, however (at age 19), she was admitted to our hospital because of severe headache and disturbance of consciousness. CT and MRI scans of her head revealed obstructive hydrocephalus caused by enlargement of the cerebellar tumors (Fig. 1B).
Ventricular drainage was performed immediately. Four weeks later, during which time she underwent cerebral angiography, among other examinations, the largest tumor was removed and histologically diagnosed as a hemangioblastoma. Ophthalmological examinations, abdominal CT, and MRI of the cervical cord also revealed retinal angiomas (Fig. 2A) in the right eye, cystic lesions in the pancreas and the left kidney, and a cystic tumor in the cervical cord (Fig. 2B). After removal of the cerebellar tumor, the patient's headaches resolved, but she suffered from hoarseness caused by palsy of the glossopharyngeal and vagal nerves.

\section{Molecular diagnosis}

To identify the VHL mutation in this nuclear family, genomic DNA was isolated from all four members and from the brain tumor of patient 3. Three coding exons of the VHL gene were analyzed in the germline of patient 3 by direct sequencing, using polymerase chain reaction (PCR) primers designed to amplify nine overlapping segments covering the entire coding region and exon-intron boundaries (Table 1), according to procedures described previously (Kurose et al. 1998). The genomic DNA of this patient

Fig. 1A,B. T1-weighted magnetic resonance images of the head of patient 3 obtained after injection of gadolinium-diethylene triamine pentaacetic acid (Gd-DTPA). A Magnetic resonance imaging (MRI) at age 14 revealed at least three enhanced lesions in the cerebellum. They were very small and represented no mass effect. B MRI at age 19. One of the lesions in the right cerebellar hemisphere was enlarged and cysts had developed within it
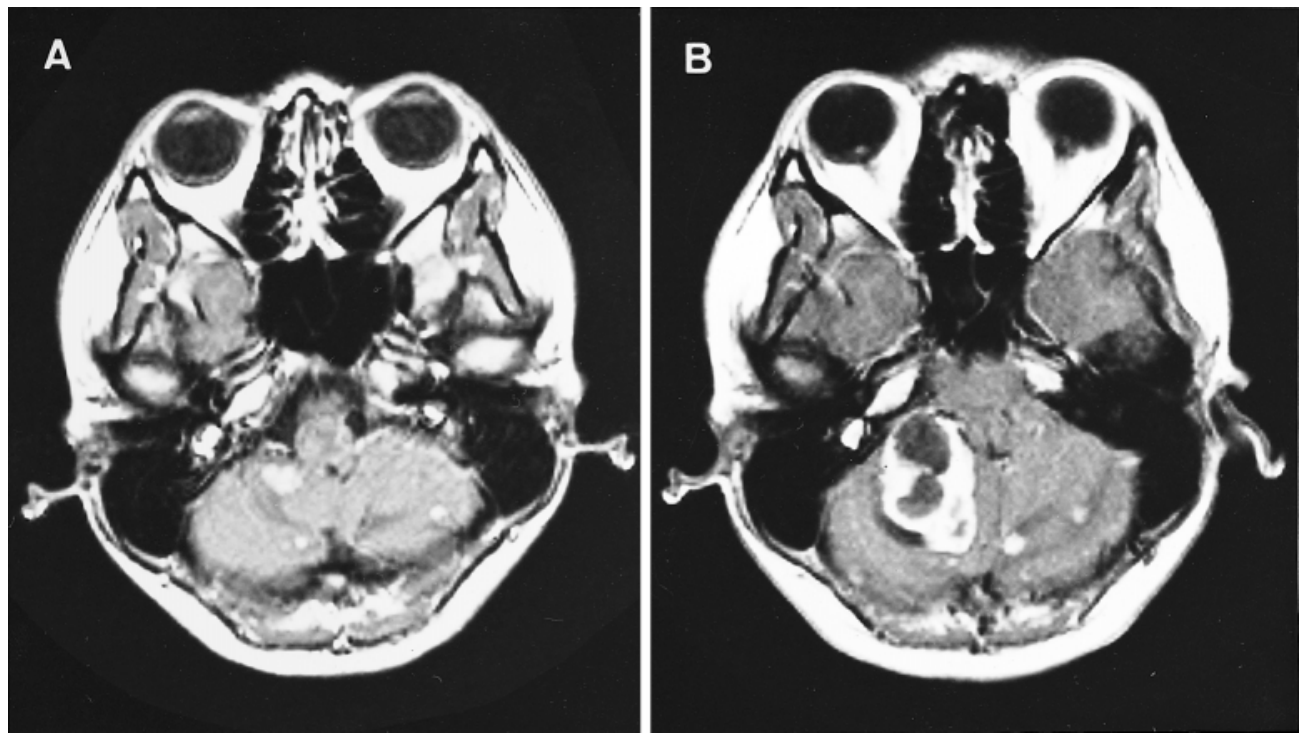

Table 1. Sequence of $V H L$ primers used for sequencing and RFLP analysis

\begin{tabular}{llll}
\hline No. & Forward primers & Reverse primers & Exon \\
\hline 1 & AGTGGAAATACAGTAACGAGTTG & TGCGCGCTCCCGAGTCG & 1 \\
2 & GAGCGCGTTCCATCCTCTAC & TCGGCCTCGTCCCAGTTCTCC & 1 \\
3 & GGTGGTCTGGATCGCGGAG & TCCTCCGGGCCGGACTCTTC & 1 \\
4 & AGGAGTCGGGCGCCGAGG & ATACGGGCAGCACGACGCG & 1 \\
5 & TCGGTGAACTCGCGCGAGC & CTATCGTCCCTGCTGGGTCG & 1 \\
6 & CCAGCCACCGGTGTGGCTC & TCCGTCAACATTGAGAGATGG & 2 \\
7 & GCTTCTGGTTAACCAAACTG & GTGGTCTATCCTGTACTTAC & 2 \\
8 & AGTCTGTCACTGAGGATTTGG & GTCAGCCGCTCCAGGTCTTC & 3 \\
9 & AGGTCGCTCTACGAAGATCTG & GTATCTAGATCAAGACTCATCAG & 3
\end{tabular}

VHL, von-Hippel-Lindau; RFLP, restriction fragment length polymorphism 
Fig. 2A,B. von-Hippel-Lindau (VHL)-related extra-cranial lesions of patient 3 . A O, ithalmoscopic photogram of the retina of tile right eye shows an angioma with a proliferative membrane, near the optic disc. B T1-weighted MRI of the cervical cord obtained after injection of Gd-DTPA revealed a cystic lesion with a highly enhanced nodule
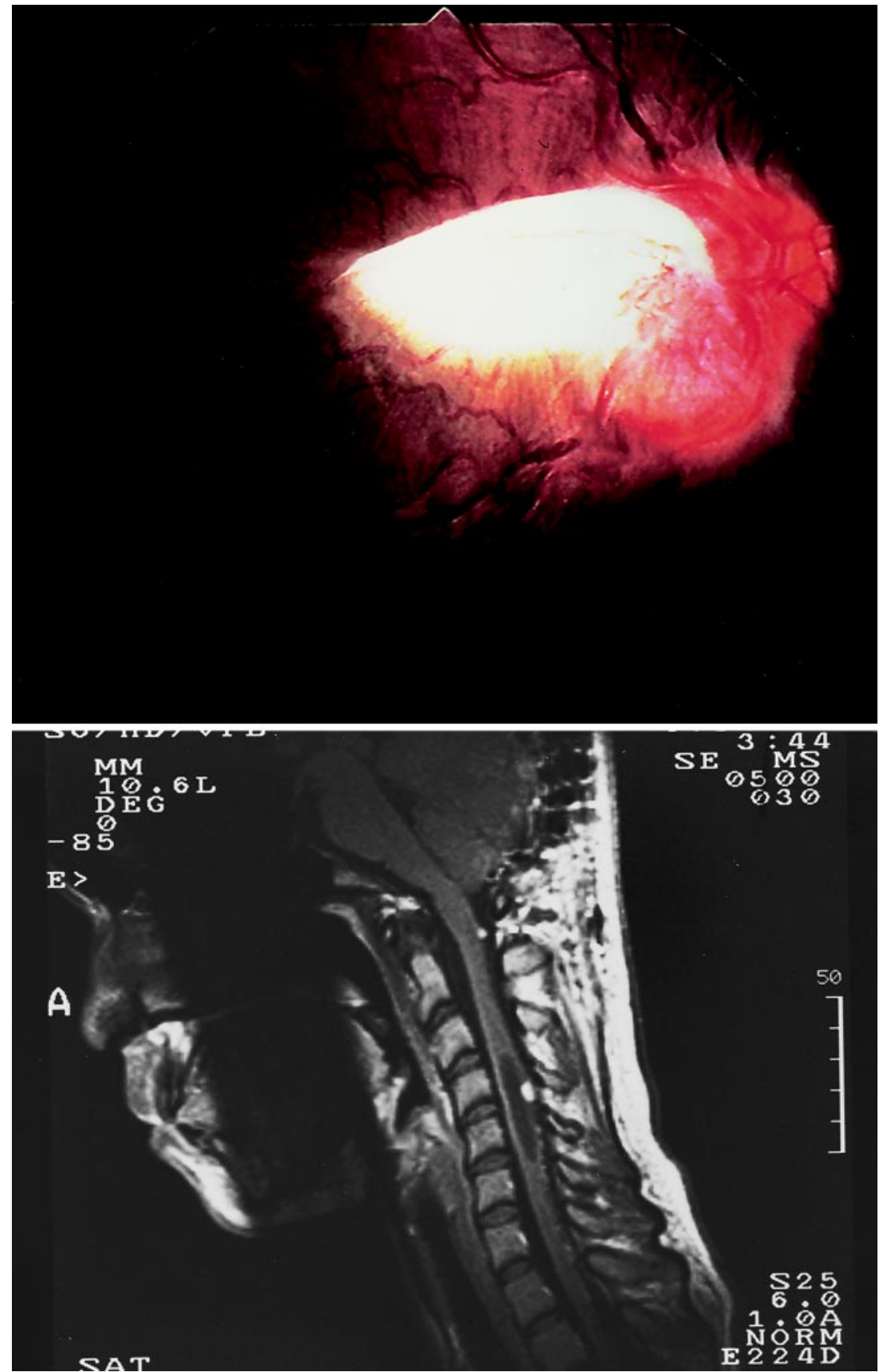

revealed heterozygosity at the second nucleotide of codon 157 (CCC-to-CTC; Fig. 3A). The C-to-T transition led to the substitution of Leu for Pro at amino acid residue 157 on the affected allele.

Since this mutation abolished a recognition site for $B g / \mathrm{I}$, we attempted genetic diagnosis of all members of the family by digesting PCR products with this endonuclease and subjecting them to agarose-gel electrophoresis and ethidium bromide staining. $B g /$ I digestion cleaved a 192-bp PCR segment into 122-bp and 70-bp fragments in the wild-type (CCC) allele, while the 192-bp band remained in the mutant (CTC) allele. The results of genetic diagnosis in this family are shown in Fig. 3B, which shows that the Pro157Leu VHL mutation was present in both of the other affected members of the family.

\section{Discussion}

A salient feature of the VHL syndrome is the variety of its clinical phenotypes. VHL pedigrees show various combinations of lesions, and such lesions generally occur sequentially rather than at the same time. The phenotypic variety seems to derive from different alterations in the VHL gene; certain symptoms appear be associated with specific muta- 
A

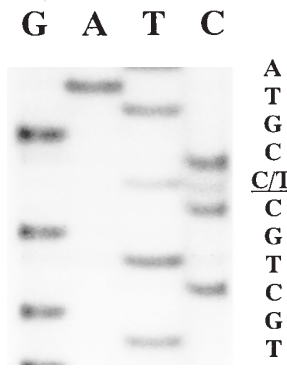

Fig. 3. A Sequence analysis of the $V H L$ gene in the tumor of patient 3 indicated heterozygosity for CCC (normal) and CTC (mutant) alleles at codon 157. B Restriction fragment length polymorphism analysis by $B g / I$ digestion. The normal allele generated 122-bp and 70-bp fragments; the mutant allele was undigested (192-bp fragment). Lane 3, father: homozygosity for the normal allele; lanes 1,2,4, and 5: heterozygosity for normal and mutant alleles in affected mother and both daughters. $G$ and $T$, germline and tumor DNAs of patient 3, respectively. Pedigree shows unaffected father (open square) and affected mother and two daughters (closed circles)

tions (Crossey et al. 1994b; Kishida et al. 1994; Chen et al. 1995; Glavac et al. 1996). The present report describes a Japanese VHL family in which two affected members manifested acute hydrocephalus as a consequence of cerebellar hemangioblastomas. We detected a Pro157Leu missense mutation of the $V H L$ gene in the genomic DNA of all three affected members.

Shuin and colleagues (Clinical Research Group for VHL in Japan, 1995) reported that the spectrum of VHL mutations in Japanese patients had features not seen in Western patients. For example, no nonsense mutations were observed in the Japanese study group, although a Pro157Leu mutation was described in a single patient; that patient had a retinal angioma but no tumors in brain, kidney, or adrenal glands. The same Pro157Leu mutation had been reported in two Caucasian VHL families as well, in whom cerebellar hemangioblastoma was the most frequent lesion associated with the mutation ( 9 of 12 affected individuals), while retinal angioma and renal cell carcinoma each occurred in 6 of the 12 patients. No pheochromocytomas, pancreatic cysts, or complications such as hydrocephalus were recorded.

In the Japanese VHL family presented here, retinal angioma and cerebellar hemangioblastoma were present in all three affected members, and two of them developed pancreatic cysts and the complication of acute hydrocephalus. One daughter had cystic tumors in the kidney and the cervical cord. Although a detailed haplotype study is yet to be done, the independent occurrence of the same missense mutation in four apparently unrelated Japanese and Caucasian VHL families suggests that proline at residue 157 may be critical for maintenance of the normal function of the VHL protein.

Sequencing of the $V H L$ gene from the cerebellar hemangioblastoma of patient 3 (Fig. 3A,B) indicated that the wildtype allele had not been lost in the tumor. Although contamination with non-tumorous cells can mask loss of a normal allele, inactivation mechanisms other than allelic loss, such as subtle mutation or hypermethylation, could have occurred to render the wild-type allele inactive (Herman et al. 1994; Tse et al. 1997).

Identification of the $V H L$ gene has opened the door to pre-symptomatic diagnosis by means of mutational analysis in VHL families. In the family examined here, mutational analysis of three affected members revealed a C-to-T substitution at the second nucleotide of codon 157, a substitution that happened to abolish a $B g / I$ cleavage site. Therefore we would be able to exploit restriction fragment length polymorphism (RFLP) analysis by $B g / \mathrm{I}$ digestion to predict whether members of the extended families of patients with this mutation do or do not carry a predisposition to VHL tumors. Relatives who are shown to be carriers of the mutant allele could be monitored by thorough examinations to forestall life-threatening complications such as occlusive hydrocephalus or renal cell carcinoma.

Systematic screening and early detection of tumors can indeed reduce morbidity and mortality from VHL disease (Seizinger et al. 1988). Up to $25 \%$ of patients with hemangioblastomas in the central nervous system actually have the VHL syndrome (Neumann et al. 1989), so individuals with those tumors should be examined for germline mutations in the VHL gene. In addition, VHL patients must be followed clinically to enable early detection of renal cell carcinoma, a life-threatening lesion (Maher et al. 1990). As some hemangioblastomas in the central nervous system require emergency treatment, early identification of such tumors in the relatives of VHL patients could avoid those situations as well (Neumann et al. 1989). Continuing efforts to expand mutational analysis and to establish a detailed description of VHL phenotypes in different populations would lead to a more thorough understanding of this tumor syndrome in the near future, and provide patients with early intervention.

Acknowledgements Supported by special Grants-in-Aid for Cancer Research and "Genome Science" from the Ministry of Education, Science, and Culture of Japan; and by a Research Grant for Cancer Research from the Ministry of Health and Welfare of Japan.

\section{References}

Chen F, Kishida T, Yao M, Hustad T, Glavac D, Dean M, Gnarra J, Orcutt M, Duh F, Glenn G (1995) Germline mutations in the von Hippel-Lindau disease tumor suppressor gene: correlations with phenotype. Hum Mutat 5:66-75

Clinical Research Group for VHL in Japan (1995) Germline mutations in the von Hippel-Lindau disease (VHL) gene in Japanese VHL. Hum Mol Genet 4:2233-2237

Crossey P, Maher E, Jones M, Richards F, Latif F, Phipps M, Lush M, Foster K, Tory K, Green J (1993) Genetic linkage between von HippelLindau disease and three microsatellite polymorphisms refines the localization of the VHL locus. Hum Mol Genet 2:279-282

Crossey P, Foster K, Richards F, Phipps M, Latif F, Tory K, Jones M, Bentley E, Kumar R, Lerman M (1994a) Molecular genetic investigations of the mechanism of tumourigenesis in von Hippel-Lindau disease: analysis of allele loss in VHL tumours. Hum Genet 93:53-58 Crossey P, Richards F, Foster K, Green J, Prowse A, Latif F, Lerman 
M, Zbar B, Affara N, Ferguson-Smith M (1994b) Identification of intragenic mutations in the von Hippel-Lindau disease tumour suppressor gene and correlation with disease phenotype. Hum Mol Genet 3:1303-1308

Duan D, Pause A, Burgess W, Aso T, Chen D, Garrett K, Conaway R, Conaway J, Linehan W, Klausnert RD (1995) Inhibition of transcription elongation by the VHL tumor suppressor protein. Science 269:1402-1406

Glavac D, Neumann H, Wittke C, Jaenig H, Masek O, Streicher T, Pausch F, Engelhardt D, Plate K, Hofler H (1996) Mutations in the VHL tumor suppressor gene and associated lesions in families with von Hippel-Lindau disease from central Europe. Hum Genet 98:271-280

Herman J, Latif F, Weng Y, Lerman M, Zbar B, Liu S, Samid D, Duan D, Gnarra J, Linehan W (1994) Silencing of the VHL tumor-suppressor gene by DNA methylation in renal carcinoma. Proc Natl Acad Sci USA 91:9700-9704

Hosoe S, Brauch H, Latif F, Glenn G, Daniel L, Bale S, Choyke P, Gorin M, Oldfield E, Berman A (1990) Localization of the von Hippel-Lindau disease gene to a small region of chromosome 3 . Genomics 8:634-640

Kibel A, Iliopoulos O, DeCaprio J, Kaelin WJ (1995) Binding of the von Hippel-Lindau tumor suppressor protein to Elongin B and C. Science 269:1444-1446

Kishida T, Yao M, Chen F, Orcutt M, Lerman M, Zbar B (1994) A novel donor splice site mutation associated with two mRNAs in von Hippel-Lindau disease. Hum Mol Genet 3:1191-1192

Kley N, Whaley J, Seizinger B (1995) Neurofibromatosis type 2 and von Hippel-Lindau disease: from gene cloning to function. Glia 15:297-307

Kurose K, Bando K, Fukino K, Sugisaki Y, Araki T, Emi M (1998) Somatic mutations of the PTEN/MMAC1 gene in 15 Japanese endometrial cancers: evidence for inactivation of both alleles. Jpn J Cancer Res 89:842-848

Maher E, Yates J, Ferguson-Smith M (1990) Statistical analysis of the two stage mutation model in von Hippel-Lindau disease, and in sporadic cerebellar haemangioblastoma and renal cell carcinoma. J Med Genet 27:311-314

Maher E, Bentley E, Yates J, Latif F, Lerman M, Zbar B, Affara N, Ferguson-Smith M (1991) Mapping of the von Hippel-Lindau disease locus to a small region of chromosome $3 p$ by genetic linkage analysis. Genomics 10:957-960

Melmon K, Rosen S (1964) Lindau's disease: review of the literature and study of a large kindred. Am J Med 36:595-617

Neumann H, Eggert H, Weigel K, Friedburg H, Wiestler O, Schollmeyer P (1989) Hemangioblastomas of the central nervous system. A 10-year study with special reference to von Hippel-Lindau syndrome. J Neurosurg 70:24-30

Seizinger B, Rouleau G, Ozelius L, Lane A, Farmer G, Lamiell J, Haines J, Yuen J, Collins D, Majoor-Krakauer D (1988) Von Hippel-Lindau disease maps to the region of chromosome 3 associated with renal cell carcinoma. Nature 332:268-269

Seizinger B, Smith D, Filling-Katz M, Neumann H, Green J, Choyke P, Anderson K, Freiman R, Klauck S, Whaley J (1991) Genetic flanking markers refine diagnostic criteria and provide insights into the genetics of Von Hippel Lindau disease. Proc Natl Acad Sci USA 88:2864-2868

Tory K, Brauch H, Linehan M, Barba D, Oldfield E, Filling-Katz M, Seizinger B, Nakamura Y, White R, Marshall F (1989) Specific genetic change in tumors associated with von Hippel-Lindau disease. $\mathrm{J}$ Natl Cancer Inst 81:1097-1101

Tse J, Wong J, Lo K, Poon W, Huang D, Ng H (1997) Molecular genetic analysis of the von Hippel-Lindau disease tumor suppressor gene in familial and sporadic cerebellar hemangioblastomas. Am J Clin Pathol 107:459-466

Yao M, Latif F, Orcutt ML, Kuzmin I, Stackhouse T, Zhou FW, Tory K, Duh FM, Richards F, Maher E, Laforgia S, Huebner K, Le Paslier D, Linehan M, Lerman M, Zbar B (1993) von Hippel-Lindau disease: identification of deletion mutations by pulsed-field gel electrophoresis. Hum Genet 92:605-614 